\title{
Design and study of MIMO systems studied
}

\author{
Bouamama Réda Sadouki ${ }^{1}$, Mouhamed Djebbouri ${ }^{2}$ \\ ${ }^{1}$ (Electronics Department, University of Sidi Bel Abbès Telecom Laboratory LTTNS, ALGERIA, \\ ${ }_{2}^{2}$ (Electronics Department, Professor, University of Sidi Bel Abbès Telecom Laboratory LTTNS, ALGERIA,
}

\begin{abstract}
In this research, we are interested in a telecommunications system MIMO (multiple input multiple output) spatial multiplexing in OFDM context. ) .

The objective of this work is to study and improve the transmission and reception of this MIMO (Multiple Input Multiple Output) systems technology in a multi -carrier OFDM context (Orthogonal Frequency Division Multiplex), which enables a frequency-selective channel into multiple non-selective channels. The combination of MIMO and OFDM allows exploiting the benefits of the two methods: the strength of binding on frequency-selective channels for OFDM and robustness on uncorrelated in space for the MIMO channel coding.

For different configurations of multi- antenna systems SISO, SIMO and MIMO, a comparative study is made between them.

This study shows that the MIMO configuration associated with the OFDM technique provides a significant performance improvement compared to other case studies.

Keywords: Multiple Input Single Output ( MISO ) orthogonal frequency division multiplexing (OFDM), Single Input Multiple Output ( SIMO ) v Single Input Single Output, Signal to Noise Ratio (SNR), bit error rate (BER) , Multiple-Input Multiple-Output (MIMO).
\end{abstract}

\section{Introduction}

The study of multiple antenna systems generally designated by MIMO (Multiple- Input Multiple - Output) systems has become the focus of much research. In the conventional configuration of MIMO systems, antennas are collocated on terminals, source and destination. Among the first to take an interest in the study of MIMO systems Telatar and Foschini [14], [15], have revitalized this research motivating a greater number of researchers.

The main observation is that, when the number of transmitting antennas and the number of receive antennas increases, the system capacity increases. Theoretically, the increase of the capacity is proportional to the minimum between the number of transmitting antennas and the number of receiving antennas. Thus, if we increase in the parallel number of transmitting antennas and the number of receiving antennas, the spectral efficiency of the system then increases linearly.

The multi-carrier term covers many acronyms (OFDM Orthogonal frequency division multiplexing). This type of modulation is advantageous when the inter-symbol interference are important in the transmission channel, which is the case when the channel is called frequency-selective .Furthermore, the selectivity phenomenon is aggravated by the presence of multiple paths due to reflections in a mobile radio environment as a channel. This problem is even more important than the transmitted bit rates increase as the frequency required to carry information at very high speed.

The multicarrier modulations can meet this challenge by using subcarriers insensitive to multipath and selectivity so easy to equalize.

The OFDM is a type of multi-carrier modulation in which subcarriers are orthogonal simplifying modulators / demodulators that are effectively realized using FFT.

\section{Mathematical Models And Simulation}

\subsection{Introduction}

we propose in this study using the MATLAB environment, simulations related to MIMO -OFDM configurations. Having shown theoretically in this work the influence of the number of antennas transmitters and receivers on the spectral efficiency called by abuse here capacity and bit error rate BER called, we conducted a simulation to visualize performance reception signals in terms of capacity and error rate by introducing spatial diversity.

And with the following assumptions: Each subchannel is a Rayleigh channel, the Doppler effect is not taken into account in modeling the channel Aditif The noise is considered white Gaussian noise ( AWGN ) 
power transmission Pt is imposed by relevant power sources ( and also hardware), Nt and $\mathrm{Nr}$ are the number of antennas transmitter receiver set respectively .

$\mathrm{M}$ the number of subcarriers, $\mathrm{T}_{\mathrm{u}}$ and $\mathrm{T}_{\mathrm{g}}$ represent the useful symbol time and the time of the guard interval.

\subsection{Transmission Capacity}

This portion of the simulation is interested in studying the impact of the number of antennas on the flow curves. This in order to observe the interest of spatial diversity used with the following parameters $(\mathrm{T}=4 \mu$ $\mathrm{s}, \mathrm{T}_{\mathrm{g}}=0.8 \mu \mathrm{s}$ ) each manipulates the subchannel are totally uncorrelated considered while reducing the levels of power, the gains in terms of bit rates are very useful material.

Once laid the foundations of information theory, we are able to calculate the capacity of a physical transmission channel. The channel capacity is a measure of the maximum amount of information that can be transmitted and received on a channel with a negligible probability of error. If we represent the input and output of a channel without memory random variables and $\boldsymbol{x} \boldsymbol{y}$ respectively, the ability of a defined [1] as the maximum mutual information between $\boldsymbol{x}$ e t $\boldsymbol{y}$ channel:

$\mathrm{c}=\max _{(\mathrm{px})} \mathrm{I}(\mathrm{x}, \mathrm{y})$

We show this influence by presenting plots of capacity curves $\mathrm{C}=\mathrm{f}$ (SNR) based on the number of antennas for transmitters and receivers.

\subsubsection{Canal SISO-OFDM}

\section{Is given by:}

$$
\mathrm{C}=\log _{2}\left(1+\gamma_{\mathrm{R}}\right) \mathrm{bit} / \mathrm{s} / \mathrm{Hz}
$$

It increases slowly, depending on the $\log 1+\gamma_{\mathrm{R}}$. When the SNR is high, a gain of $3 \mathrm{~dB} \gamma_{\mathrm{R}}$ not provides an increase in capacity than a bit per second per hertz (bit / s / Hz).

The capacity increases slowly versus log

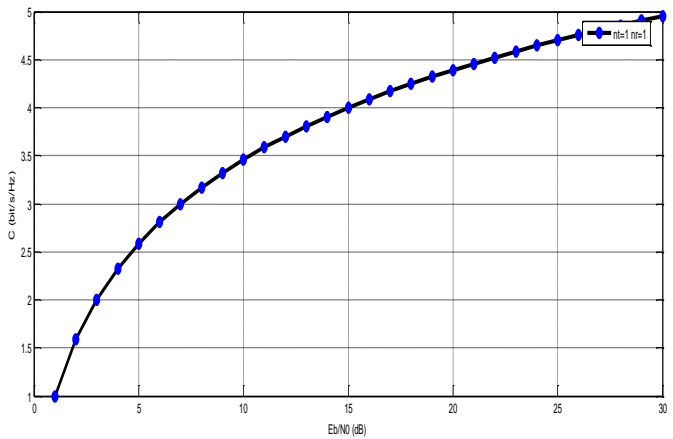

Figure 1: Capacity of SISO-OFDM system based on the SNR

\subsubsection{Canal SIMO-OFDM}

a channel SIMO (Single Input, Multiple Output) is a multi-antenna system Conventional realizing, for example, the formation of conventional route reception. Its capacity is given by:

$$
\mathrm{c}=\log _{2}\left(1+\gamma_{\mathrm{R}} * \mathrm{~N}_{\mathrm{r}}^{2}\right)(\mathrm{bit} / \mathrm{s} / \mathrm{Hz}
$$

$$
\begin{aligned}
& \gamma_{\text {instant }}=\frac{E_{s}\left(\sum_{i=1}^{n R}\left\|h_{i}\right\|^{2}\right)^{2}}{N_{0}\left(\sum_{i=1}^{n R}\left\|h_{i}\right\|^{2}\right)} \\
& \gamma_{\text {instant }}=\frac{E_{s}\left(\sum_{i=1}^{n R}\left\|h_{i}\right\|^{2}\right)}{N_{0}} \\
& \gamma_{\text {instant }}=\sum_{i=1}^{n R}\left\|h_{i}\right\|^{2} \cdot \gamma \\
& C=\log _{2}\left(1+\sum_{i=1}^{n R}\left\|h_{i}\right\|^{2} \cdot \gamma\right) \text { Bite } / \mathrm{s} / \mathrm{Hz}
\end{aligned}
$$




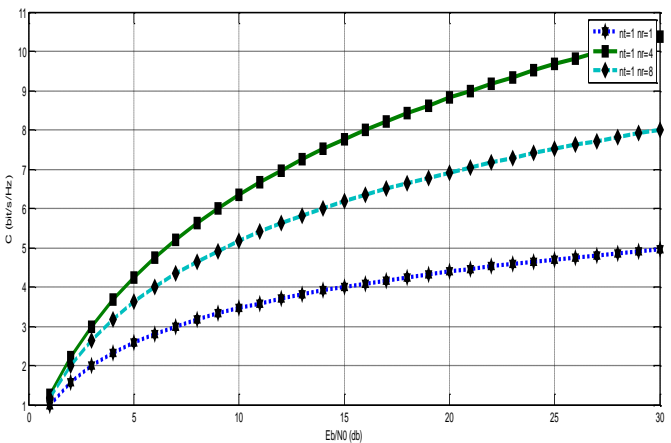

Figure 2: Capacity of SIMO-OFDM system based on the SNR.

\subsubsection{Simulation of a MIMO-OFDM channel}

For a MIMO channel with power ${ }^{\mathrm{p}_{0}} / \mathrm{N}_{\mathrm{T}}$ on each transmitter, the capacity is developed as

$\mathrm{y}=\mathrm{Hx}+\mathrm{b}$

With:

$$
\mathrm{x}=\left[\mathrm{x}_{1}, \mathrm{x}_{2}, \ldots \ldots, \mathrm{x}_{\mathrm{nT}}\right]^{\mathrm{T}}
$$

and

$$
\mathrm{y}=\left[\mathrm{y}_{1}, \mathrm{y}_{2}, \ldots \ldots, \mathrm{y}_{\mathrm{nR}}\right]^{\mathrm{T}}
$$

The matrix $\mathrm{H}$ can be decomposed in a unique decomposition into three matrices: Where $\mathrm{U}$ and $\mathrm{V}$ are unitary matrices $\mathrm{UU}^{* \mathrm{t}}=\mathrm{VV}^{* \mathrm{t}}, \mathrm{U}^{* \mathrm{t}}$ and $\mathrm{V}^{* \mathrm{t}}$ is the conjugate matrix transposed matrices $\mathrm{V}$ and $\mathrm{U}$ respectively) and $\mathrm{D}$ is a diagonal matrix whose nonzero elements are the values of $\mathrm{H}$, $\left(\mathrm{D}=\operatorname{diag}\left(\gamma_{\mathrm{i}}\right)\right)$ matrices $\mathrm{U}, \mathrm{D}$ and $\mathrm{V}$ are the respective sizes $\mathrm{N} \times \mathrm{m}, \mathrm{m} \times \mathrm{m}, \mathrm{m} \times \mathrm{M} ;$ avec $\mathrm{m}=\min (\mathrm{N}, \mathrm{M})$, that is to say:

$\mathrm{H}=\mathrm{U} \times \mathrm{D} \times \mathrm{V}^{\mathrm{H}} ; \mathrm{m}=\min (\mathrm{M}, \mathrm{N})$

By inserting in the chain of transmission appropriate stages preceding and post coding, we can prove the independence of MIMO channel $m$ system as follows:

$$
\begin{aligned}
& \tilde{y}=U^{*} \cdot Y \\
& \tilde{y}=U^{*} \cdot\left(U D V^{*} \cdot X+n\right) \\
& \tilde{y}=U^{*} \cdot\left(U D V^{*} \cdot V \widetilde{X}+n\right) \\
& \left.\tilde{y}=U^{*} \cdot\left(U D V^{H}\right) \cdot V \widetilde{X}+U^{H} n\right) \\
& \tilde{y}=\underbrace{U^{*} \cdot\left(U D V^{H}\right)}_{\text {D }} \cdot V \widetilde{X}+\underbrace{\left(U^{H} n\right)}_{\widetilde{n}} \\
& \tilde{y}=\operatorname{diag}\left(\gamma_{i}\right) \cdot \widetilde{X}+\widetilde{n} \\
& \tilde{y}=D \cdot \widetilde{X}+\widetilde{n}
\end{aligned}
$$

power $\left.\mathrm{P}_{\mathrm{T}} / \mathrm{N}\right)$ :

$$
\begin{aligned}
& \text { The capacity of a subchannel (for a transmitted powe } \\
& \mathrm{C}_{\mathrm{i}}=\log _{2}\left(1+\frac{\mathrm{P}_{\mathrm{T}}}{\mathrm{N}}\left|\gamma_{\mathrm{i}}\right|^{2}\right)
\end{aligned}
$$

The capacity of a MIMO system as the previous one:

$$
\begin{aligned}
& \mathrm{C}=\sum_{\mathrm{i}=1}^{\mathrm{m}} \mathrm{C}_{\mathrm{i}} \\
& \mathrm{C}=\sum_{\mathrm{i}=1}^{\mathrm{m}} \log _{2}\left(1+\frac{\mathrm{P}_{\mathrm{T}}}{\mathrm{N}}\left|\gamma_{\mathrm{i}}\right|^{2}\right)
\end{aligned}
$$

Especially if we assume $\lambda \mathbf{i}=\lambda$, then the capacity is written:

$$
\mathrm{C}=\mathrm{m} \cdot \log _{2}\left(1+\frac{\mathrm{p}}{\mathrm{N}}\left|\gamma_{\mathrm{i}}\right|^{2}\right)
$$

This capability is usually written as follows:

$$
\mathrm{C}=\log \operatorname{det}\left(\mathrm{I}_{\mathrm{M}}+\left(1+\frac{\mathrm{p}}{\mathrm{N}} \mathrm{HH}^{\mathrm{T}}\right)\right)
$$

$$
\text { Cases without knowledge }
$$
of the channel (no CSI) same power allocated to different transmitters (strategy $B L A S T$ )

$$
\mathrm{C}=\log _{2}\left(\operatorname{det}\left[\mathrm{I}_{\mathrm{N}_{\mathrm{r}}}+\frac{\mathbb{7}_{\mathrm{R}}}{\mathrm{N}_{\mathrm{t}}} \mathrm{HH}^{*}\right]\right) \mathrm{Bit} / \mathrm{s} / \mathrm{Hz}
$$




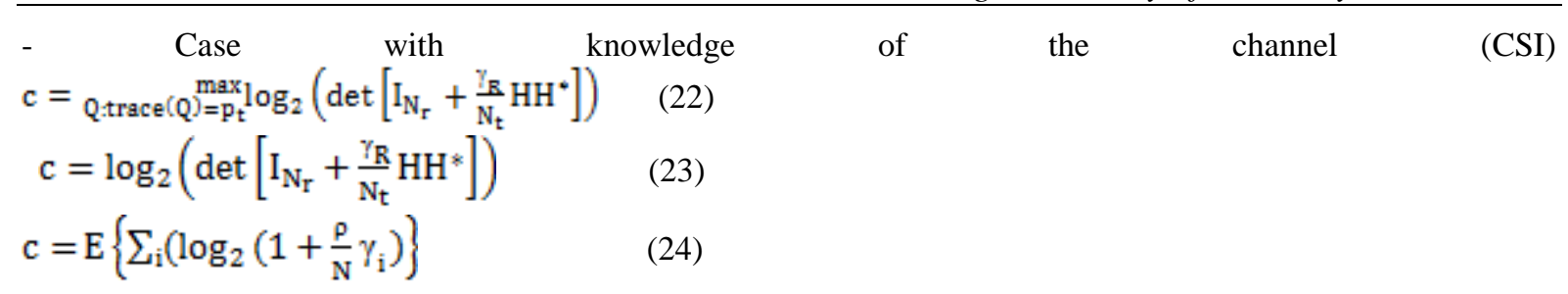

The formula for defining the ability of paper as a prior reference [ 1 ], [2 ], [3 ], [ 4 ], [5 ] .

\section{Comparison between SISO-OFDM, MIMO and OFDM, SIMO-OFDM}

we observe a marked increase in capacity for the MIMO configuration with respect to SISO and SIMO case. The advantage in capacity of MIMO systems is mainly due to the exploitation of multipath. Firstly they enable the receiver to distinguish the different transmitting antennas, and thus transmit simultaneously several symbols.

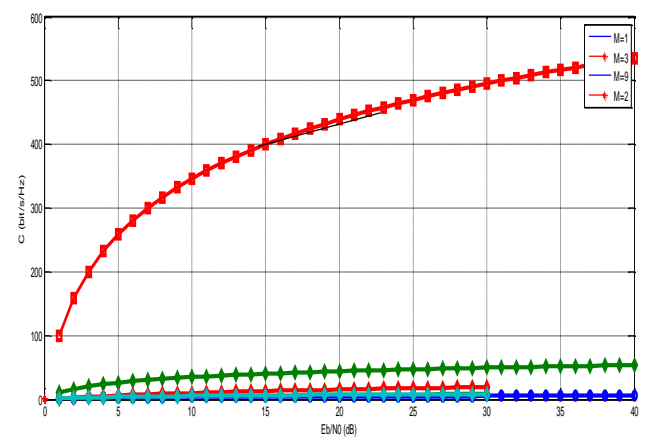

Figure 3: Comparison between SISO-OFDM, SIMO-OFDM, MIMO-OFDM

3/4 In the SISO case $(\mathrm{Nt}=1$ and $\mathrm{Nr}=1)$ capacity ranges from 1 to $4.5 \mathrm{bps} / \mathrm{Hz}$ approximately. It remains low and increases slowly with Eb/NO, which illustrates the limitations of SISO transmissions. Despite the current techniques, which allow making the most of a SISO channel capacity is a terminal that cannot be exceeded and a multi-antenna system, even under exploited, get better performance.

$3 / 4$ The two examples SIMO $(\mathrm{Nt}=1$ and $\mathrm{Nr}=3$ and $\mathrm{Nr}=9)$ show the upper bounds of spatial multiplexing without treatment. The transition to three receive antennas saves $3 \mathrm{bps} / \mathrm{Hz}$ compared to SISO, which is not very important, especially at high Eb/N0.

With $\mathrm{Nr}=9$ gain is about $2 \mathrm{bps} / \mathrm{Hz}$, which is not for another four antennas. As for SISO systems capacity increases slowly, which remains the main limitation of SIMO systems, including high SNR. 3/4 MIMO two examples have the same total number of antennas that SIMO systems, so as to facilitate comparisons $(\mathrm{Nt}+\mathrm{Nr}=4$ and 8). For a SNR of $0 \mathrm{~dB}$, the MIMO system with $(\mathrm{Nt}=2$ and $\mathrm{Nr}=2$ is almost equivalent to the capacity SIMO system with four antennas). MIMO capacity then increases much faster to finish with a gain of more than $50 \%$ at $21 \mathrm{~dB}$ SNR. Exactly the same comments apply to the SIMO and MIMO systems to eight antennas.

\section{Study of performance}

We show this effect for a curve plot of the error rate as a function of energy E0/N0 that is the ratio between the energy per bit and one-sided noise spectral density BER $=f($ SNR) for different numbers of antenna transmitters and receivers.

\subsection{Error rate with SISO -OFDM}

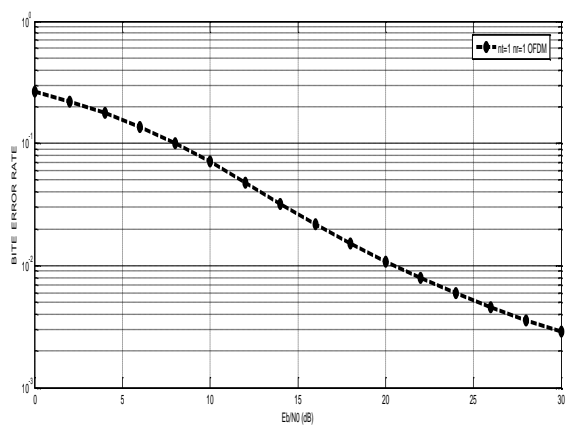

Figure 4: Error rate per symbol according to the SNR for a SISO-OFDM channel, M $=512$ (subcarriers). 
The simulated channel is a Rayleigh channel that is to say that the transmitted signal is affected by fading and white Gaussian noise Aditif (AWGN).

At low SNR, the AWGN and fading are the main disturbance signal which provides large values of BER.

\subsection{Error rate with SIMO-OFDM}

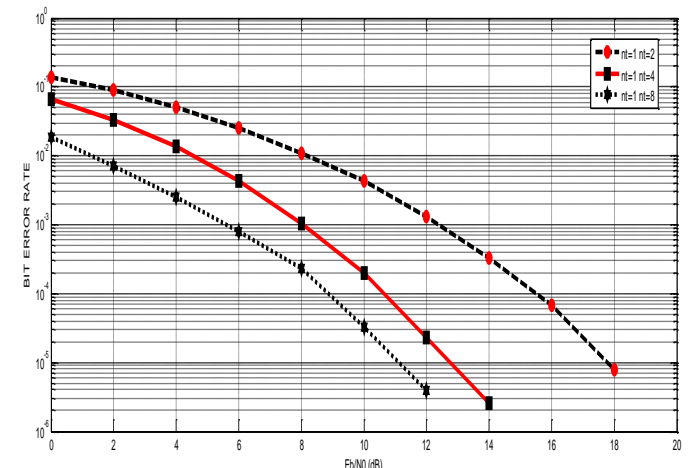

Figure 5: Error rate per symbol as a function of SNR for a SIMO-OFDM channel M = 512 (subcarriers). The antenna reception multiplicity allows the use of combination techniques replicas to combat distortion and fading suffered by the signal during transmission. The BER decreases with increasing the number of antennas and combining the OFDM technique for increasing SNR.

\subsection{Error rate with MIMO-OFDM}

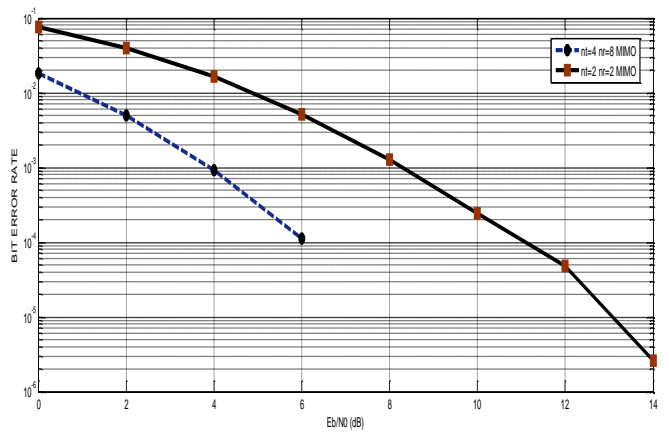

Figure 6: per symbol error rate as a function of SNR for a MIMO-OFDM channel. $\mathrm{M}=512$ (subcarriers). The use of space diversity transmission and reception entails application algorithms reception. Which assumes that the number of receptors is at least as large as the number of transmitters for low BER and therefore an optimal signal reception?

In summary, we can say that the more diversity order increases, the number of subcarriers is large so to eliminate the phenomenon of interference between symbol, the error rate will decline with the growth of SNR. In this figure we present a comparison between SISO, SIMO and MIMO For a SNR equal to 5 dB the BER is approximately:

$\cdot 10^{-0.15}$ for a SISO channel.

$\cdot 10^{-2.2}$ for a SIMO channel.

$\cdot 10^{-3.8}$ for a MIMO channel.

4.4 Study of the influence of the number of subcarriers on the signal quality

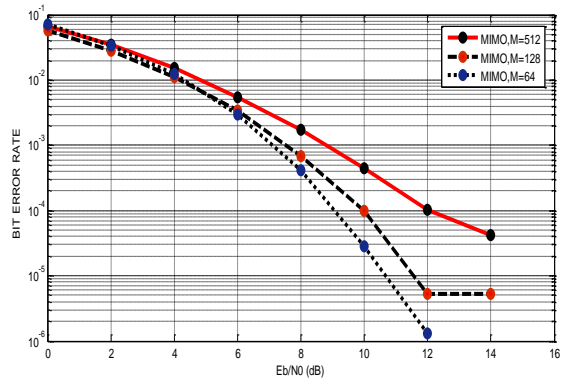

Figure 7: Influence of the number of subcarriers on the performance of the MIMO system for $\mathrm{Nt}=2, \mathrm{Nr}$ $=4$ 
We find that performance improves significantly when the number of subcarriers increases.

\section{Conclusion}

Our work is based on consideration of the capacity of the propagation channel, and the minimization of the bit error rate (or symbols).

According to our results, we can see that the capacity increases indefinitely with the number of transmitters and receivers for increasing SNR.

Furthermore, the MIMO -OFDM combination provides better quality of signal reception by eliminating the selectivity of the channel and reducing the phenomenon of inter-symbol interference and thus most of the diversity order increases the higher the rate of error decreases, the signal reception is optimal (high SNR)

We found that the capacity increases indefinitely with the number of transmitting and receiving antennas for signal reports on the growing noise and more about diversity, the higher the bit error rate or symbol is minimized and the quality signal reception is better. These performances are greatly improved by the combination of MIMO with OFDM.

\section{References}

[1] C. E. Shannon. A Mathematical theory of communication. Bell Systems Technical Journal, 27:379-423 and 623-656, July and October 1948.

[2] R. Gautier, G. Burel, J. Letessier, and O. Berder. Blind estimation of scrambler offset using encoder redundancy. In Proceedings of IEEE Asilomar Conference on Signals, Systems and Computers, volume 1, pages 626-630, Pacific Grove (CA), USA, 2002.

[3] John G. Proakis. Digital communications. McGraw-Hill, Third Edition, 1995.[A.F.Mol2005]: A. F. Molisch: "Wireless communications", chap 20, Ed Wiley 2005.

[4] H. Bölcskei and A. J. Paulraj. The Communications Handbook, chapter Multiple-input multiple-output (MIMO) wireless systems. CRC Press, 2001.

[5] B. Le Floch, M. Alard, and C. Berrou. Coded orthogonal frequency division multiplex. IEEE Proceedings, 83(6):982-996, 1995.

[6] G. Burel, C. Bouder, and O. Berder. Detection of direct sequence spread spectrum transmissions without prior knowledge. In Proceedings of IEEE Global Telecommunications Conference (Globecom), volume 1, pages 236-239, San Antonio (TX), USA, November 2001.

[7] O. Berder, C. Bouder, and G. Burel. Identification of frequency hopping communications. In Proceedings of WSEAS Conference on Circuits, Systems, Communications and Computers (CSCC), pages 3851-3856, Vouliagmeni, Greece, July 2000.

[8] R. G. Vaughan. Polarization diversity in mobile communications. IEEE Transactions on Vehicular Technology, 39:177-186, August 1990.

[9] V. Erceg, L. Greenstein, S. Tjandra, S. Parkoff, A. Gupta, B. Kulic, A. Julius, and R. Bianchi. An empirically based path loss model for wireless channels in suburban environments. IEEE Journal on Selected Areas in Communications, 17(7):1205-1211, July 1999.

[10] W. C. Jakes. Microwaves mobile communications. McGraw-Hill, New-York, 1982.

[11] A. Mansour, C. Jutten, and P. Loubaton. Adaptive subspace algorithm for blind separation of independent sources in convolutive mixture. IEEE Transactions on Signal Processing, 48(2):583-586, February 2000.

[12] A. Wittneben. Basestation modulation diversity for digital simulcast. In Proceedings of the IEEE Vehicular Technology Conference (VTC'91), pages 848-853, May 1991.

[13] N. Seshadri and J. H. Winters. Two signaling schemes for improving the error performance of frequency-division-duplex (FDD) transmission systems using transmitted antenna diversity. International Journal of Wireless Information Networks, 1(1):49-59, January 1994.

[14] I. E. Telatar. Capacity of multi-antenna Gaussian channels. European Transactions on Telecommunications, 10(6):585-595, 1999.

[15] G. J. Foschini and M. J. Gans. On limits of wireless communications in a fading environment when using multiple antennas. Wireless Personal Communications, 6(3):311-335, march 1998

[16] H. B"olcskei and A.J. Paulraj, "Space-Frequency coded broadband OFDM Systems," IEEE WCNC 2000, Chicago (Il.), USA, September 2000.

[17] J. Yang and S. Roy, "On joint transmitter and receiver optimization for multiple-input-multiple-output (MIMO) transmission systems," IEEE Transactions on Communications, Vol. 42, No. 12, pp. 3221-3231, December 1994. 